\title{
NÁUSEAS Y VÓMITOS POSTOPERATORIOS
}

\section{Arias González, Xiomara ${ }^{1}$; Quirós Rodríguez, Adriana ${ }^{2}$ y Rodríguez Miranda, Roberto ${ }^{3}$}

1Médico Especialista en Anestesiología y Recuperación, Hospital Nacional de Niños, San José, Costa Rica. ${ }^{2}$ Médico Especialista en Anestesiología y Recuperación, Hospital San Juan de Dios, San José, Costa Rica. ${ }^{3}$ Médico Especialista en Anestesiología y Recuperación. Residente de Algología, Instituto Nacional de Ciencias Médicas y Nutrición Salvador Zubirán, México D.F. México.

Resumen: Las náuseas y los vómitos postoperatorios (NVPO) se incluyen entre las experiencias más desagradables que pueden presentar los pacientes sometidos a cirugía bajo anestesia general. Su incidencia es 20 a 30\%, pero puede alcanzar hasta un $80 \%$ en pacientes de alto riesgo sin profilaxis.

La etiología de NVPO es multifactorial. Aunque se cuenta con varias opciones terapéuticas para su prevención y tratamiento, ningún medicamento disponible, por sí solo, es capaz de abolirlas. El abordaje multimodal ha demostrado ser una estrategia eficaz para prevenir o mitigar las mismas.

La presente revisión repasa la incidencia y las bases fisiopatológicas de NVPO. Además, presenta las herramientas necesarias para identificar a los pacientes que tienen mayor riesgo de presentar NVPO con el objetivo de establecer el plan anestésico idóneo para cada uno de ellos. Conjuntamente, describe los fármacos disponibles más utilizados para profilaxis antiemética y terapia de rescate.

Palabras clave: Náuseas, vómitos, postoperatorio, antieméticos. 


\section{POSTOPERATIVE NAUSEA AND VOMITING}

Abstract: Postoperative Nausea and Vomiting (PONV) is included among the most unpleasant experiences a patient can witness following general anesthesia. Its incidence ranges from 20 to $30 \%$, but can reach up to $80 \%$ in high risk patients receiving no prophylaxis.

The etiology of PONV is multifactorial. Even though many therapeutic options are available for its prevention and treatment, no single intervention is capable of abolishing PONV. However, the multimodal approach has been proven to be an effective aid.

The present review covers the incidence and pathophysiology of PONV, as well as present the tools needed to identify high risk patients with the purpose of establishing the best anesthetic plan for them. Finally, we describe the medications commonly used for PONV prophylaxis and rescue treatment.

Key words: Nausea, vomiting, postoperative, antiemetics

\section{INTRODUCCIÓN}

Las NVPO se presentan con frecuencia en pacientes que son sometidos a cirugía bajo anestesia general. Esta complicación ha estado presente por décadas, a pesar de las mejoras en las técnicas quirúrgicas y anestésicas, y de los avances en la farmacología antiemética[1,2].

Las NVPO complican la recuperación de la cirugía y son motivo de profundo malestar para el paciente, además, generan atrasos en los egresos de las Unidades de Cuidado Posanestésico (UCPA) y readmisiones hospitalarias, produciendo elevación en los costos de atención médica. Las NVPO son la causa más importante de estancia hospitalaria prolongada tras cirugía ambulatoria, tanto en niños como en adultos [3].

\section{FISIOPATOLOGÍA}

El vómito se produce a través de una serie de cambios autonómicos complejos que operan en el cerebro a nivel del bulbo raquídeo desencadenados por diferentes factores fisiológicos y patológicos.

El control primario de náuseas y vómitos proviene del centro del vómito, que es una región neuroanatómica localizada en la superficie dorsal del bulbo raquídeo hacia la porción final del IV ventrículo y presenta un número de vías neuronales interrelacionadas [4].

Existen 5 vías aferentes primarias involucradas en la estimulación del centro de náusea y vómito: la zona gatillo quimiorreceptora (ZGQ), la vía vagal de la mucosa gastrointestinal, las vías neuronales del sistema vestibular, las vías aferentes reflejas de la corteza cerebral y las aferencias del mesencéfalo [4].

Todas estas señales son mediadas a través de sistemas de receptores y neurotransmisores que incluyen el sistema serotoninérgico, dopaminérgico, histaminérgico, colinérgico y neuroquininérgico. Los antieméticos para profilaxis y tratamiento de las NVPO actúan por el bloqueo de uno o más de estos receptores: 5- $\mathrm{HT}_{3}$ de serotonina, $\mathrm{D}_{2}$ de dopamina, $\mathrm{H}_{1}$ de histamina, colinérgico muscarínico y $\mathrm{NK}_{1}$ de neuroquinina $[5,4,6]$.

\section{FACTORES DE RIESGO}

\section{Relacionados con el paciente}

Género femenino: Se estima que las mujeres tienen tres veces más riesgo de presentar NVPO que los hombres [7]. Se ha sugerido que las variaciones en los niveles de gonadotropinas sería la causa de la alta incidencia de ésta complicación en éste género, debido a que se ha demostrado que no hay diferencia en la incidencia de NVPO entre el género masculino y 
femenino en la etapa de preadolescencia y en pacientes mayores de 80 años [7].

No fumado: Cohen y colaboradores encontraron que los pacientes no fumadores tenía 1.8 veces más probabilidad de presentar NVPO que los pacientes fumadores. Esto podría estar relacionado con la inducción de enzimas del citocromo P450 (CYP450), las cuales contribuyen al metabolismo de agentes volátiles y otros anestésicos conllevando a una disminución de las NVPO $[8,9]$.

Historia de NVPO: Un episodio anterior de NVPO, es en los análisis de regresión, un factor de riesgo muy importante, para que en el próximo procedimiento anestésico se presente de nuevo esta complicación [10].

Historia de cinetosis: Es un término definido como malestar, debilidad, fatiga, náusea e incluso vómito, producido por la aceleración y desaceleración lineal y angular repetitivas, la historia de tal concepto es, según los análisis de regresión logística, uno de los factores de riesgo más fuertemente asociadas al desarrollo de NVPO[10].

\section{Cuadro 1. Factores involucrados en la estimulación del centro de la náusea y vómito}

\begin{tabular}{|c|c|}
\hline Áreas que afectan el centro del vómito & Estímulos \\
\hline $\begin{array}{l}\text { Área postrema } \\
\text { ZGQ }\left(5-H_{3}, D_{2}, M_{1}, H_{1}, \mu, ~ ¿ ?\right)\end{array}$ & $\begin{array}{l}\text { - Alteraciones en sangre y LCR provocadas por fármacos, } \\
\text { radiación, otros trastornos metabólicos } \\
\text { - Movimiento, sistema vestibular, cerebelo }\end{array}$ \\
\hline $\begin{array}{l}\text { Núcleo del tracto solitario } \\
\left(5-\mathrm{HT}_{3}, \mathrm{D}_{2}, \mathrm{M}_{1}, \mathrm{H}_{1}\right)\end{array}$ & -Aferentes viscerales \\
\hline Aferentes corticales & -Nervio trigémino, orofaringe, vista, olfato \\
\hline Aferentes viscerales & $\begin{array}{l}\text {-Tracto gastrointestinal, orofaringe, corazón, pulmón, anestesia, } \\
\text { cirugía, a través de SNP (nervio vago) y SNS (dolor) }\end{array}$ \\
\hline
\end{tabular}

LCR: Líquido cefalorraquídeo. SNP: Sistema nervioso parasimpático. SNS: Sistema nervioso simpático. Tomado de referencia 48

\section{Relacionados con la técnica anestésica}

Anestesia regional: La incidencia de NVPO después de técnicas anestésicas regionales, con o sin sedación es baja. Este es el resultado encontrado en el meta análisis realizado por Liu y colaboradores, que comparó la anestesia regional (bloqueo periférico del nervio o bloqueo neuroaxial) contra la anestesia general en la cirugía ambulatoria [11].
Propofol: Se ha asociado este medicamento con disminución significativa en la incidencia de NVPO. Estudios han mostrado que es efectivo a corto plazo cuando se brinda en infusión continua para el mantenimiento de la anestesia en pacientes con riesgo para NVPO $[12,27,43,44]$.

Agentes halogenados: Apfel y colaboradores concluyeron que los agentes volátiles fueron la principal causa de emesis en el postoperatorio precoz, siendo su efecto dependiente del tiempo de exposición

Revista electrónica publicada por el Departamento de Farmacología de la Escuela de Medicina de la Universidad de Costa Rica, 2060 San José, Costa Rica. ${ }^{\circledR}$ All rights reserved. Licensed under a Creative Commons Unported License. 
y similar para los distintos halogenados (sevoflurano, isoflurano y enflurano) [2].

Óxido nitroso: Paul Myles condujo un estudio en Australia donde comparó un grupo de óxido nitroso $\left(70 \% \mathrm{~N}_{2} \mathrm{O}, \mathrm{O}_{2} 30 \%\right)$ con un grupo de aire enriquecido con oxígeno $\left(80 \% \mathrm{O}_{2}, \mathrm{~N}_{2} 20 \%\right)$ en el cual se evidenció más NVPO en los pacientes que recibieron óxido nitroso en el transoperatorio [1]. El óxido nitroso estimula directamente el centro del vómito e interactúa con receptores opioides y dopaminérgicos, además, aumenta la presión del oído medio, produce distensión intestinal y disminución de la motilidad intestinal [13].

Opioides: La acción emetizante de los opioides se debe a la estimulación directa de la ZGQ, prolongación del vaciamiento gástrico, reducción de la motilidad gastrointestinal y sensibilización del sistema vestibular. En el postoperatorio los opioides duplican el riesgo de NVPO, en este período el riesgo es dosis dependiente [14].

\section{Factores relacionados con el procedimiento quirúrgico}

La manipulación intestinal durante la cirugía y la disección vascular esplácnica asociada resulta en cierta isquemia intestinal. El estrés de la cirugía mayor per se estimula el sistema nervioso simpático y puede reducir sustancialmente el flujo sanguíneo esplácnico. $\mathrm{La}$ isquemia intestinal desencadena un ascenso importante de 5- $\mathrm{HT}_{3}$ y otros factores eméticos [15]. Durante la manipulación intestinal, podría liberarse serotonina de las células enterocromafines de la mucosa, y ésta podría actuar sobre la ZGQ tanto a través de la circulación sistémica como estimulando las vías aferentes de los nervios vago [16].

Existen procedimientos quirúrgicos que se asocian con mayor riesgo para desarrollar NVPO, la incidencia varía según el tipo y la duración de la cirugía. Se estima que cada 30 minutos de incremento del tiempo quirúrgico, aumenta el riesgo de NVPO en un $60 \%$ a partir del riesgo basal $[17,18,19]$.

\section{Otros factores}

No está claro si el uso de sonda orogástrica o nasogástrica para descompresión gástrica, disminuye la presentación de NVPO, dado que la documentación existente al respecto no es concluyente $[16,19]$.
En los pacientes obesos parece estar incrementado el riesgo emético debido a una acumulación de anestésicos volátiles liposolubles en el tejido adiposo, que al liberarse lentamente a la circulación prolongarían la duración de sus efectos secundarios. Pero, los datos disponibles hasta la fecha para este subgrupo de pacientes son insuficientes y contradictorios [20].

\section{Cuadro 2. Procedimientos quirúrgicos frecuentemente asociados con NVPO}

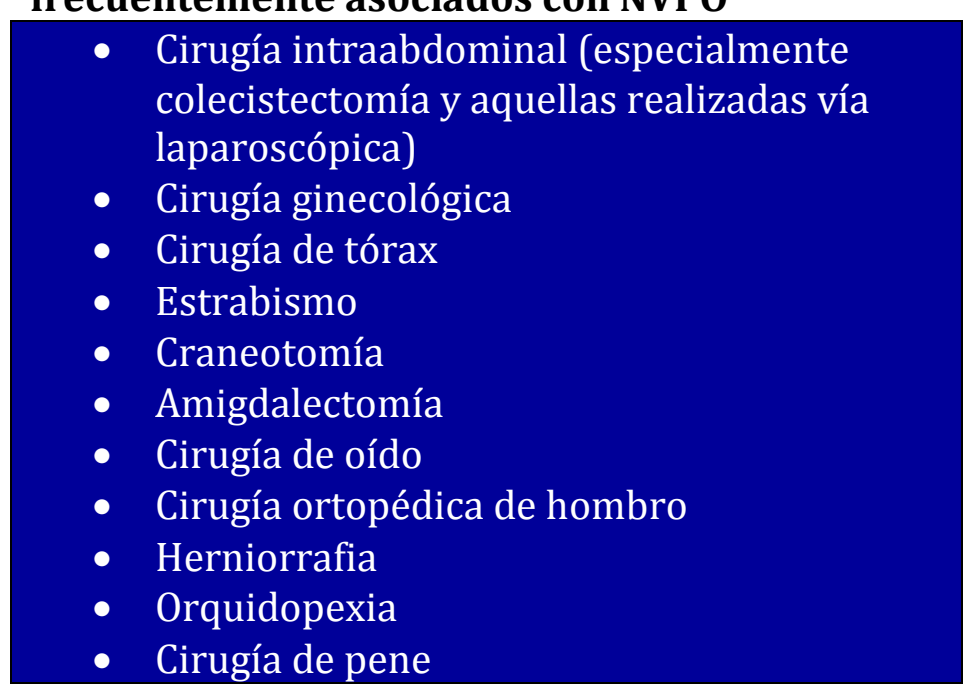

Tomado de referencia 18.

Pusch y colaboradores han establecido que la inestabilidad hemodinámica durante la inducción y el mantenimiento anestésicos puede desempeñar un papel importante en el desarrollo de las NVPO, las pacientes con variaciones de más del 35\% de la presión arterial sistólica (PAS) con respecto a la de base, presentaron mayor incidencia de NVPO [21].

En sus respectivos trabajos, Magner, Haentjens y Holte, encontraron que la incidencia de NVPO fue significativamente menor cuando se utilizaba fluido terapia perioperatoria. Esta impide la hipoperfusión intestinal y previene isquemia intestinal y liberación de serotonina, la cual juega un papel importante en la génesis de la emesis. Además, no encontraron diferencias en el uso de coloides y cristaloides $[22,23,24]$.

\section{Sistemas de puntaje predictores de NVPO}

Por su seguridad predictiva y facilidad de aplicación existe el consenso generalizado entre los 
expertos de que los mejores indicadores son los propuestos por Apfel y Koivuranta [25,26,27].

La evaluación de Apfel, la más reconocida, incluye cuatro variables y asigna un punto por cada una. La suma total de puntos se correlaciona con la probabilidad de presentar NVPO. La evaluación Koivuranta incluye cinco variables asigna un punto por cada una [26].

Algunos clasifican el riesgo para NVPO según la presencia de cada uno de estos factores así: 0 a 1 bajo riesgo, 2 factores como de moderado riesgo y de 3 o más como de alto riesgo [27].

Se considera que los pacientes de moderado y alto riesgo para NVPO son los que se benefician de una profilaxis antiemética, la cual es más efectiva cuando se combinan dos o más agentes con diferentes mecanismos de acción [12,17, 25, 28, 43].

\section{MEDICAMENTOS ANTIEMÉTICOS}

Para su estudio, los fármacos antieméticos pueden agruparse según el tipo de receptor sobre el que ejercen su acción, ya sea colinérgico muscarínico, dopaminérgico, histaminérgico, serotoninérgico o neuroquininérgico. En el cuadro 4 se encuentran anotados, los fármacos antieméticos que se comercializan en Costa Rica y sus dosis habituales cuando se utilizan para prevención y tratamiento de NVPO. Todos ellos, excepto el granisetron, ondasetron y aprepitant, se encuentran disponibles en la Caja Costarricense del Seguro Social como terapia de NVPO. El uso de los tres fármacos mencionados, dentro de la institución, está limitado al tratamiento de náuseas y vómitos asociados a quimioterapia.

Cuadro 3. Puntuación de riesgo de Apfel et al

\begin{abstract}
Factores de Riesgo
Puntos
\end{abstract}

Suma total de puntos

Probabilidad de NVPO

(\%)

Género Femenino

1

0

10

No fumador

1

1

21

Historia de NVPO o Mareo

1

2

39

Uso esperado de opioides

1

3

61

postoperatorios

Total

$0-4$

4

78

Tomado de referencia 47

\section{Medicamentos anticolinérgicos}

Dentro de este grupo, el medicamento que ha demostrado mayor utilidad es la escopolamina, que actúa bloqueando la transmisión colinérgica del núcleo vestibular hacia los centros superiores del SNC y de la formación reticular hacia el centro del vómito. Es efectiva para prevenir NVPO cuando el procedimiento quirúrgico se asocia con estímulo vestibular [29].

\section{Antagonistas de receptores de histamina}

La difenhidramina, dimenhidrinato, hidroxicina y prometazina son fármacos antihistamínicos disponibles en la Caja Costarricense del Seguro Social.

Como antiemético se utiliza principalmente el dimenhidrinato. Este medicamento bloquea receptores $\mathrm{H}_{1}$ de histamina en la NTS, en el centro del vómito y el sistema vestibular [7]. Se utiliza efectivamente para tratar la cinetosis y la NVPO posterior a cirugía del oído medio. Sin embargo, la sedación es un efecto adverso común, por lo que no es popular como profilaxis de NVPO [30].

Revista electrónica publicada por el Departamento de Farmacología de la Escuela de Medicina de la Universidad de Costa Rica, 2060 San José, Costa Rica. ${ }^{\circledR}$ All rights reserved. Licensed under a Creative Commons Unported License. 


\section{Antagonistas de receptores de dopamina}

Actúan inhibiendo receptores $\mathrm{D}_{2}$ sobre la zona quimiorreceptora. Dentro de este grupo, los más estudiados como antieméticos son la metoclopramida y el droperidol.

La metoclopramida es una benzamida que tiene propiedades procinéticas, aumenta el vaciamiento gástrico y acorta el tiempo de tránsito intestinal. En altas dosis puede bloquear los receptores de serotonina en la zona quimiorreceptora [7]. Ha sido utilizada durante décadas para evitar las NVPO. Sin embargo, varios meta análisis han mostrado que la dosis IV de 10 mg usada comúnmente, no es efectiva para prevención de NVPO [31,32]. Por otra parte, estudios recientes apoyan que una dosis de metoclopramida de 25 a $50 \mathrm{mg}$ sí disminuye la incidencia de NVPO, pero se asocia con hipotensión, taquicardia y otros efectos adversos $[33,34]$.

Dentro del grupo de las butirofenonas se destaca el droperidol, que tiene características antieméticas bien probadas, incluso en dosis pequeñas $[7,33]$. Se recomienda dar al final de la cirugía y en la dosis efectiva más baja para reducir al mínimo la sedación potencial, que es su principal efecto adversos [34].

La Administración de Drogas y Alimentos (FDA, por sus siglas en inglés) recibió informes de arritmias severasy muertes asociadas con el uso del droperidol, por lo que advierte que está contraindicado en pacientes con prolongación del QT, ya que esta condición se ha asociado con taquicardia ventricular tipo Torsades de Pointes. De la misma forma, Agencias de Control de Medicamentos de varios países europeos, han publicado advertencias sobre la seguridad del empleo crónico de dosis altas orales de droperidol, por lo que se retiró esta presentación. Para el tratamiento de NVPO, el droperidol se utiliza en forma parenteral y dosis bajas, de 0,625 a $2.5 \mathrm{mg}$, han demostrado ser efectivas [7,33]. Sin embargo, queda sin aclararse el riesgo de arritmias, y como resultado, su utilización ha disminuido [51]. Diversos estudios, han demostrado que la prolongación del QTc es similar después del uso del droperidol o de antagonistas $5-\mathrm{HT}_{3}[36,37]$.

La FDA recomienda, que cuando se utiliza, debe confirmarse previamente, mediante electrocardiograma (ECG), la ausencia de prolongación del QT corregido (QTc) y después de su administración, debe supervisarse continuamente el ECG por 2 a 3 horas
[35]. El intervalo QT se mide desde el inicio del complejo QRS hasta el final de la onda T. Se utiliza la medida promedio de 3 a 5 derivaciones, escogiendo aquellas donde sea mayor. El QTc puede calcularse mediante la fórmula de Bazett: QTc $=Q T / \sqrt{R R}$. Se considera prolongado cuando es mayor a $450 \mathrm{~ms}$ en adultos masculinos o mayor a $470 \mathrm{~ms}$ en mujeres adultas [50]. Hay que tener especial precaución si existen factores de riesgo para Torsades de Pointes inducida por drogas, como hipokalemia, bradicardia, insuficiencia cardiaca congestiva, terapia con digitálicos, conversión reciente desde un ritmo de fibrilación atrial, síndromes subclínicos de QT largo, polimorfismo de canales iónicos, hipomagnesemia severa, o combinación con otras drogas asociadas a prongación del QT, por ejemplo, quinidina, procainamida, sotalol, amiodarona, claritromicina, eritromicina, antidepresivos tricíclicos, litio, clorpromazina, haloperidol o metadona, especialmente si las concentraciones del fármaco son altas o la velocidad de infusión intravenosa es rápida [49].

\section{Antagonistas de receptores de serotonina $5-\mathrm{HT}_{3}$}

Los antagonistas de los receptores de serotonina subtipo $5-\mathrm{HT}_{3}$, que se encuentran en mayor proporción en el NTS y en la ZGQ, se consideran efectivos tanto en la profilaxis como en el tratamiento de NVPO [7]. De hecho, la Sociedad para Anestesia Ambulatoria (SAMBA, por sus siglas en inglés) publicó en 2010 guías de manejo de NVPO, donde los recomienda como agentes de primera línea para la prevención de NVPO en pacientes de alto riesgo [37].

Dentro de este grupo se encuentran el dolasetrón, granisetrón, ondansetrón, ramosetrón, tropisetrón y palonosetrón, entre otros. La evidencia disponible sobre el tiempo óptimo de administración de una dosis mínima efectiva de estos fármacos es aún controversial. Mientras la información de prescripción dada por las casas farmacéuticas, indica aplicarlos al inicio de la cirugía; SAMBA en sus guías del 2010, recomienda darlos al final del procedimiento $[37,38]$.

\section{Corticoesteroides}

Su mecanismo de acción no está claro, se supone que es por disminución de los niveles de neurotransmisor disponible y reducción de la liberación de prostaglandina $\mathrm{E}$, con su respectiva función antiinflamatoria o estabilizadora de membrana tanto a nivel periférico como central [7]. 
La dexametasona ha demostrado ser efectiva en el tratamiento y prevención de la emesis inducida por quimioterapia, así como en la profilaxis de NVPO, especialmente cuando es administrada previo a la inducción de la anestesia [37,39].

\section{Antagonistas de receptores de neuroquinina.}

Corresponden a una nueva clase de antieméticos que bloquean el receptor de neuroquinina $\left(\mathrm{NK}_{1}\right)$ en el área postrema y la acción de la sustancia $\mathrm{P}$, que puede ser liberada de las células enterocromafines del estómago y el intestino o de neuronas sensoriales [7].

No se encontraron meta análisis para valorar profilaxis o tratamiento de NVPO, pero sí estudios multicéntricos que comparan el aprepitant, un fármaco de este grupo, con el ondansetrón, obteniendo resultados similares en cuanto a incidencia de NVPO [40]. Una consideración es que este medicamento debe administrarse antes de la cirugía, ya que sólo se dispone de presentación oral [41].

\section{Cuadro 4. Sitios de acción, dosis usuales, y efectos adversos de la selección de los medicamentos} antieméticos en adultos

\begin{tabular}{|c|c|c|c|c|c|c|}
\hline Antiemético & $\begin{array}{l}\text { Sitio de } \\
\text { acción }\end{array}$ & $\begin{array}{l}\text { Dosis usual en } \\
\text { adultos }\end{array}$ & $\begin{array}{l}\text { Duración } \\
\text { de acción }\end{array}$ & $\begin{array}{l}\text { Efectos } \\
\text { adversos }\end{array}$ & Comentarios & $\begin{array}{l}\text { NNT* } \\
\text { global }\end{array}$ \\
\hline Droperidol & $\overline{D_{2}}$ & $0.625-1.25 \mathrm{mg}$ iv & $\begin{array}{l}\text { Hasta 12- } \\
24 \mathrm{~h}\end{array}$ & $\begin{array}{l}\text { Sedación, mareos, } \\
\text { ansiedad, } \\
\text { hipotensión, } \\
\text { SEP** }\end{array}$ & $\begin{array}{l}\text { Monitoreo de QT, > } \\
\text { efectivo para náusea } \\
\text { que vómito }\end{array}$ & 5 \\
\hline Metoclopramida & $\mathrm{D}_{2}$ & $\begin{array}{l}25 \text { o } 50 \mathrm{mg} \text { iv para } \\
\text { profilaxis }\end{array}$ & Hasta $6 \mathrm{~h}$ & $\begin{array}{l}\text { Sedación, } \\
\text { hipotensión y } \\
\text { SEP** }\end{array}$ & $\begin{array}{l}\text { Dosis }<25 \mathrm{mg} \text { iv no } \\
\text { son efectivas }\end{array}$ & 10 \\
\hline Dimenhidrinato & $\mathrm{H}_{1}, \mathrm{M}_{1}$ & $\begin{array}{l}1-2 \mathrm{mg} / \mathrm{kg} \text { o } 50- \\
100 \mathrm{mg} \mathrm{iv}, \mathrm{im}\end{array}$ & $6-8 \mathrm{~h}$ & $\begin{array}{l}\text { Sedación, boca } \\
\text { seca, visión } \\
\text { borrosa, mareos, } \\
\text { RAO }\end{array}$ & & 5 \\
\hline Granisetrón & $5-\mathrm{HT}_{3}$ & $5 \mu \mathrm{g} / \mathrm{kg}-1 \mathrm{mg} \mathrm{iv}$ & Hasta $24 \mathrm{~h}$ & $\begin{array}{l}\text { Cefalea, mareo, } \uparrow \\
\text { enzimas } \\
\text { hepáticas }\end{array}$ & $\begin{array}{l}\text { Más efectivo para } \\
\text { vómito que náusea }\end{array}$ & 5 \\
\hline Ondansetrón & $5-\mathrm{HT}_{3}$ & $4 \mathrm{mg}$ iv & Hasta $24 \mathrm{~h}$ & $\begin{array}{l}\text { Cefalea, mareo, } \uparrow \\
\text { enzimas } \\
\text { hepáticas }\end{array}$ & $\begin{array}{l}\text { Más efectivo para } \\
\text { vómito que náusea }\end{array}$ & 5 \\
\hline Dexametasona & ningún & $4 \mathrm{mg}$ iv & Hasta $24 \mathrm{~h}$ & $\begin{array}{l}\text { Prurito vaginal, } \\
\text { irritación anal }\end{array}$ & $\begin{array}{l}\text { La simple dosis es } \\
\text { bien tolerada. }\end{array}$ & 4 \\
\hline Aprepitant & $\mathrm{NK}_{1}$ & $40 \mathrm{mg}$ vo & Hasta $24 \mathrm{~h}$ & $\begin{array}{l}\text { Cefalea y } \uparrow \\
\text { enzimas } \\
\text { hepáticas }\end{array}$ & $\begin{array}{l}\text { Dar } 3 \mathrm{~h} \text { antes de } \\
\text { inducción }\end{array}$ & $7-11$ \\
\hline
\end{tabular}

*NNT: Número necesario a tratar, parámetro de eficacia de reducción de riesgo. ${ }^{* *}$ SEP: síntomas extrapiramidales. Tomado de referencia 5 .

Revista electrónica publicada por el Departamento de Farmacología de la Escuela de Medicina de la Universidad de Costa Rica, 2060 San José, Costa Rica. ${ }^{\circledR}$ All rights reserved. Licensed under a Creative Commons Unported License. 


\section{Combinaciones y terapia multimodal}

En la prevención y tratamiento de NVPO se utilizan combinaciones de agentes antieméticos, debido a que hay al menos cuatro sistemas principales de receptores implicados en su mecanismo [42]. Además, la etiología de las NVPO es multifactorial y ningún fármaco disponible actualmente tiene efectividad cercana al $100 \%$ [43].

Entre las combinaciones más estudiadas se encuentra la de los antagonistas de $5 \mathrm{HT}_{3}$ con droperidol, dexametasona o metoclopramida. En general, las combinaciones con metoclopramida no parecen tener ningún efecto beneficioso adicional, mientras que la combinación con droperidol o dexametasona son significativamente más efectivas que la monoterapia $[7,37,43]$.

\section{Manejo profiláctico de NVPO}

En la práctica clínica para obtener un manejo efectivo de la NVPO, se debe identificar a los pacientes de riesgo, mantener un riesgo basal bajo y utilizar antieméticos en combinación cuando el paciente lo amerite [37].

En los pacientes con riesgo bajo de presentar NVPO, no se recomienda el uso rutinario de medicamentos en forma profiláctica, a menos que tengan una condición médica de riesgo en caso de vómito. En los pacientes con moderado riesgo se recomienda la monoterapia. Si el riesgo es alto, se utilizaría terapia combinada con agentes de diferentes clases, en forma típica, un antagonista de los receptores de $5 \mathrm{HT}_{3}$ más dexametasona [37].

\section{Cuadro 4. Estrategias de reducción del riesgo basal de NVPO y nivel de evidencia}

\begin{tabular}{|c|c|}
\hline Usar & Anestesia regional (IVA) \\
\hline Evitar & $\begin{array}{c}\text { Estímulos emetizantes: } \\
-\quad \text { Oxido nitroso (IIA) } \\
\text { - } \quad \text { Anestésicos inhalatorios (IA) } \\
\text { - } \quad \text { Etomidato y Ketamina (V) } \\
\text { - }\end{array}$ \\
\hline Minimizar & $\begin{array}{ll}\text { Uso de opioides } \\
-\quad \text { Intraoperatorios (IIA) } \\
-\quad \text { Postoperatorios (IVA) } \\
\text { Uso de neostigmina (IIA). No usar más de } 2.5 \mathrm{mg} \text { en adultos (V) }\end{array}$ \\
\hline Considerar & $\begin{array}{l}\text { Anestesia endovenosa total con propofol (IA) } \\
\text { Hidratación adecuada (IIIA) } \\
\text { Uso de oxígeno suplementario (IIIB) } \\
\text { Ansiolisis con benzodiazepinas (IIIB) }\end{array}$ \\
\hline
\end{tabular}

Tomado de referencia 44

\author{
Nivel de evidencia científica según el diseño del \\ estudio \\ I: Ensayo clínico aleatorizado, n>100 por grupo \\ II: Revisión sistemática \\ III: Ensayo clínico aleatorizado, $\mathrm{n}<100$ por grupo \\ IV: Ensayo clínico no aleatorizado, o caso clínico \\ V: Opinión de expertos
}

Fuerza de la recomendación según la opinión de los expertos
A: Buena evidencia para apoyar la recomendación
B: Suficiente evidencia para apoyar la recomendación
C: Insuficiente evidencia para apoyar la recomendación

La anestesia regional está menos asociada a NVPO que la anestesia general. Se recomienda, siempre que sea posible. Además, disminuye los requerimientos

Revista electrónica publicada por el Departamento de Farmacología de la Escuela de Medicina de la Universidad de Costa Rica, 2060 San José, Costa Rica. ${ }^{\circledR}$ All rights reserved. Licensed under a Creative Commons Unported License. 
de otros agentes como halogenados y opioides. En caso de que sea necesario utilizar anestesia general, se considera que omitir el uso de agentes inhalados, y utilizar anestesia total intravenosa con propofol, lograría evitar NVPO en pacientes de alto riesgo, incluso más que la administración de antieméticos profilácticos $[37,44]$.

Debe considerarse el abordaje multimodal del dolor perioperatorio, con el uso de anestésicos locales y antiinflamatorios no esteroideos, acetaminofén o algún neuromodulador, que provea una adecuada analgesia y disminuya la necesidad de opioides [44].

\section{Terapia de rescate de NVPO}

En contraposición con la profilaxis de NVPO, existe menos información sobre la eficacia de la terapia antiemética en pacientes con NVPO ya establecidos.

La terapia depende del empleo o no de profilaxis previa y del tipo de fármaco empleado en ella. En caso de haber utilizado ondansetrón o dexametasona como profilaxis, y aparecer NVPO, no se recomienda repetir dosis del mismo fármaco si no han pasado más de 6 horas en el caso de ondansetrón o más de 24 horas en el caso de dexametasona, desde su administración inicial [45].

Las opciones terapéuticas posibles una vez realizada la profilaxis recomendada son limitadas y el manejo de las NVPO establecidos, difícil. Es importante descartar causas tratables de náuseas y vómitos, como hipotensión arterial, hipovolemia, dolor o disminución postoperatoria de la motilidad gastrointestinal $[45,46]$.

\section{CONCLUSIONES}

La etiología de las NVPO es multifactorial. Como launiversalización de la profilaxis antiemética, no ha demostrado ser costo-efectiva, se considera que la estratificación de riesgo es fundamental para un abordaje adecuado. Para tal efecto, se puede utilizar la puntuación de riesgo de Apfel, que es fácil de recordar y ha demostrado buen poder predictivo. En los pacientes que tienen alto riesgo de presentar NVPO, su prevención no se basa sólo en el uso de fármacos antieméticos; es importante también minimizar los estímulos emetizantes. Fármacos diversos, como el droperidol, y los antagonistas $5-\mathrm{HT}_{3}$ han demostrado ser efectivos en el tratamiento y prevención de las NVPO. Sin embargo, en los últimos años, se ha cuestionado la asociación del droperidol con arritmias severas, por lo que los antagonistas $5-\mathrm{HT}_{3}$ se han ido convirtiendo en el medicamento de elección para esta entidad.

\section{REFERENCIAS}

1. Myles PS, Williams DL, Hendrata M, Anderson $H$, Weeks AM. Patient satisfaction after anaesthesia and surgery: results of a prospective survey of 10,811 patients. Br J Anaesth 2000; 84:6-10.

2. Watcha MF. Postoperative nausea and emesis. Anesthesiol Clin North Am 2002; 20:709-22.

3. Shaikh S, Chung F, ImarengiayeCh, Yung D, Berstein M. Pain, nausea, vomiting and ocular complications delay discharge following ambulatory microdiscectomy. Can J Anesth 2003; 50: 514-518.

4. Couture D, Maye J, O Brien D, Smith AB. Therapeutic modalities for the prophylactic management of postoperative nausea and vomiting. J Perianesth Nurs 2006; 21 (6):398403.

5. Gan TJ. Mechanisms underlying postoperative nausea and vomiting and neurotransmitter receptor antagonist-based pharmacotherapy CNS Drugs 2007; 21 (10): 813-833.

6. Hornby PJ. Central neurocircuitry associated with emesis. Am J Med 2001;111Suppl 8A: 106 107.

7. Kovac AL. Prevention and treatment of postoperative nausea and vomiting. Drugs 2000; 59: 213-43.

8. Whalen F, Sprung J, Burkle ChM, Schroeder DR, Warner DO. Recent Smoking Behavior and Postoperative Nausea and Vomiting. Anesth Analg 2006; 103:70-5.

9. Chimbira W, Sweeney BP. The effect of smoking on postoperative nausea and vomiting. Anaesthesia 2000; 55:540-4.

10. Islam S, Jain PN. Post-operative nausea and vomiting (NVPO): A review article. Indian J. Anaesth. 2004; 48(4): 253-258.

11. Liu SS, Strodtbeck WM, Richman JM, Wu CL. A comparison of regional versus general anesthesia for ambulatory anesthesia: A meta- 
analysis of randomized controlled trials. Anesth Analg 2005; 101:1634-1642.

12. Golembiewsky J, Tokumaru S. Pharmacological prophylaxis and management of adult postoperative/postdischarge nausea and vomiting. J Perianesth Nurs 2006; 21 (6): 385397.

13. Myles PS, Leslie K, Chan MT. Avoidance of nitrous oxide for patients undergoing major surgery: A randomized controlled trial. Anesthesiology 2007; 107:221-231.

14. Roberts GW, Bekker TB, Carlsen HH. Postoperative nausea and vomiting are strongly influenced by postoperative opioid use in a doserelated manner. Anesth Analg 2005; 101:13431348.

15. Joris JL, Poth NJ, Djamadar Am, Sessler DI, Hamoir EE, Defêchereux TR, Meurisse MR, Lamy ML. Supplemental oxygen does not reduce postoperative nausea and vomiting after thyroidectomy. Br J Anaesth 2003; 91: 857-861.

16. Wattwil M, Thörn S-E, Lövqvist A, Wattwil L, Klockhoff H, Larsson L-G, Näslund I. Perioperative gastric emptying is not a predictor of early postoperative nausea and vomiting in patients undergoing laparoscopic cholecystectomy. Anesth Analg 2002; 95: 476479.

17. Lagos C, Quezada S. Profilaxis y Tratamiento de las náuseas y vómitos postoperatorios. Rev Chil Anest, 2009; 38: 24-33.

18. Cheng CR, Sessler DI, Apfel CC. Does neostigmine administration produce a clinically important increase in postoperative nausea and vomiting? AnesthAnalg. 2005 November; 101(5): 13491355.

19. Jones JE, Tabaee A, Glasgold R, Gomillion MC. Efficacy of gastric aspiration in reducing posttonsillectomy vomiting. Arch Otolaryngol Head Neck Surg 2001; 127: 980-984.

20. Kranke P, Apfel CC, Papenfuss T. An increased body mass index is no risk factor for postoperative nausea and vomiting. A systematic review and results of original data. Acta Anaesthesiol Scand 2001; 45:160-6.

21. Apfel CC, Kranke P, Eberhart LH. Comparison of surgical site and patient's history with a simplified risk score for the prediction of postoperative nausea and vomiting. Anaesthesia 2004; 59:1078-1082.

22. Magner JJ, McCaul C, Carton E. Effect of intravenous crystalloid infusion on postoperative nausea and vomiting after gyneacological laparoscopy: comparison of 30 and $10 \mathrm{ml} / \mathrm{kg}$. Br J Anaesth 2004;93:381-385.

23. Haentjens LL, Ghoundiwal D, Touhiri K. Does infusion of colloid influence the occurrence of postoperative nausea and vomiting after elective surgery women? Anesth Analg. 2009; 108:178893.

24. Holte K, Klarskov B, Christensen DS. Liberal versus restrictive fluid administration to improve recovery after laparoscopic cholecystectomy. Ann Surg 2004; 240-892-9.

25. Van den Bosch JE, Kalkman CJ, Vergouwe Y, Van Klei WA, Bonsel GJ, Grobbee DE, et al. Assessing the applicability of scoring systems for predicting postoperative nausea and vomiting. Anaesthesia. 2005 Apr; 60(4):323-31.

26. Ebell M. Predicting postoperative nausea and vomiting. Am Fam Physician 2007; 75(10): 1537-8.

27. Gan TJ, Meyer TA, Apfel CC, Chung F, Davis PJ, Habib AS. Society for Ambulatory Anesthesia guidelines for the management of postoperative nausea and vomiting. Anesth Analg. 2007; 105: 1615-28.

28. Apfel C, Korttila K, Abdalla M, Kerger H, Turan A, Vedder I. A factorial trial of six interventions for the prevention of postoperative nausea and vomiting. N Engl J Med. 2004; 350:2441-51.

29. Golembiewsky J, Tokumaru S. Pharmacological prophylaxis and management of adult postoperative/postdischarge nausea and vomiting. J Perianesth Nurs 2006; 21 (6): 385397.

30. Kranke P, Morin AM, Roewer N. The efficacy and safety of transdermal scopolamine for the prevention of postoperative nausea and vomiting: A quantitative systematic review. Anesth Analg 2002; 95:133-143.

31. Zárate E, Corrales E, Guzmán JM, Alcalá R, Fragoso W, Soto C. La metoclopramida no disminuye la incidencia de náusea y vómito postoperatorios cuando se asocia a dexametasona en pacientes ambulatorios 
llevados a procedimientos otorrinolaringológicos. Rev Col Anest 2009; 37:13-20.

32. Kolodzie K, Apfel C. Nausea and vomiting after office-based anesthesia. Curr Opin Anaesthesiol 2009;22(4):532-8.

33. 33. Wallenborn J, Gelbrich G, Bulst D. Prevention of postoperative nausea and vomiting by metoclopramide combined with dexamethasone: randomized double blind multicentre trial. BMJ 2006; 333(7563): 313-4.

34. Murphy E. Metoclopramide also acts at serotonin receptors. Anaesth Intensive Care 2007; 35:447.

35. Buttner M, Walder B, von Elm E. Is low dose haloperidol a useful antiemetic? A meta-analysis of published and unpublished randomized trials. Anesthesiology 2004; 101:1454-6336.

36. Charbit B, Albaladejo P, Funck-Brentano C.

Prolongation of QTc interval after postoperative nausea and vomiting treatment by droperidol or ondansetron. Anesthesiology 2005; 102:10941137.

37. Le TP, Gan TJ. Update on the Management of Postoperative Nausea and Vomiting and Postdischarge Nausea and Vomiting in Ambulatory Surgery. Anesthesiology Clinics 2010; 28: 225-249.

38. Ho KY, Gan TJ. Pharmacology, pharmacogenetics and clinical efficacy of 5-hydroxytryptamine type 3 receptor antagonists for postoperative nausea and vomiting. Curr Opin Anaesthesiol 2006; 19: 606-611.

39. Warren A, King L.A review of the efficacy of dexamethasone in the prevention of postoperative nausea and vomiting. J Clin Nurs 2006; 17: 58-68.

40. Gan TJ, Apfel CC, Kovac A. A randomized, doubleblind comparison of the NK1 antagonist aprepitant versus ondansetron for the prevention of postoperative nausea and vomiting. Anesth Analg 2007; 104:1082-1089.

41.Diemunsch P, Gan TJ, Philip BK. Single-dose aprepitant vrs ondansetron for the prevention of postoperative nausea and vomiting: A randomized, double-blind phase III trial in patients undergoing open abdominal surgery. $\mathrm{Br}$ J Anaesth 2007; 99:202-211.
42. White PF. Prevention of postoperative nausea and vomiting - a multimodal solution to a persistent problem. N Engl J Med 2004;350:2511-2.

43. Habib AS, Gan TJ. Combination therapy for postoperative nausea and vomiting: a more effective prophylaxis? Ambulatory Surg 2001; 9:59-7144.

44. Bel, I. Gambús, P. Estratificación del riesgo, profilaxis y tratamiento de las náuseas y vómitos postoperatorios. Rev. Esp. Anestesiol. Reanim. 2006; 53: 301-311.

45. Scuderi PE, James RL, Harris L, Mims GR. Multimodal antiemetic management prevents early postoperative vomiting after outpatient laparoscopy. Anesth Analg 2000; 91:1408-14.

46. American Society of Anesthesiologists Task Force on Postanesthetic Care. Practice guidelines for postanesthetic care. Anesthesiology 2002;96:742-52.

47. Kranke P, Schuster F, Eberhart L. Recent advances, trends and economic considerations in the risk assessment, prevention and treatment of postoperative nausea and vomiting. Pharmacother 2007; 8 (18): 3217-323.

48. Bustos F, López S. Náuseas y vómitos postoperatorios. Manejo del paciente quirúrgico ambulatorio en atención primaria. Ergon, España, 2006; 5: 55-66.

49. Roden DM. The Long QT Syndrome (ClinicalPractice Series) N Engl J Med 2008; 358:169-176.

50. Goldenber I, Moss AJ. Long QT Syndrome. J Am Coll Cardiol 2008; 51(24): 2291-2300.

51. Gómez JI. Droperidol y arritmias cardíacas. Rev. España. Anestesiol Reanim. 2003; 50: 221-224.

\section{INFORMACION DE AUTOR:}

Roberto Rodríguez Miranda. Email: roberto rm@hotmail.com

Lugar de Trabajo: Departamento Clínica de Dolor, Instituto Nacional de Nutrición Salvador Zubirán, México DF. México. 